\title{
Clinical and microbiological characterization of Aerococcus urinae bacteraemias at Helsinki metropolitan area, Finland
}

\author{
Reetta Sihvonen ${ }^{1,2} \cdot$ Maria Turunen $^{1} \cdot$ Laura Lehtola $^{3} \cdot$ Laura Pakarinen $^{3} \cdot$ Juha O. Grönroos $^{4}$. \\ Kaisu Rantakokko-Jalava ${ }^{4}$. Anu Pätäri-Sampo ${ }^{1}$
}

Received: 5 September 2021 / Accepted: 31 January 2022 / Published online: 8 March 2022

(c) The Author(s) 2022

\begin{abstract}
Our objective was to assess the incidence of bacteraemic Aerococcus urinae cases at Helsinki metropolitan area, Finland, from a 6-year study period (2013 to 2018) and to further characterize available cases. The study evaluates the outcome of commonly used cefuroxime treatment and determinate a set of $A$. urinae in vitro antimicrobial susceptibilities for benzylpenicillin, cefuroxime, and ceftriaxone. Clinical records of $A$. urinae bacteraemic patients were reviewed retrospectively. Antimicrobial susceptibility testing was performed by disk diffusion, gradient test, and broth microdilution for 139-141 clinical A. urinae isolates. Clinical data of 72/77 patients were combined with the in vitro susceptibilities. We found an increasing number of bacteraemic $A$. urinae cases within 6 -year study period $(p=0.01)$. The patients were mainly elderly males, and all suffered from underlying conditions. A total of $27.3 \%$ of cases (21/77) showed polymicrobial blood cultures. Thirty-day mortality was $22.1 \%$. Cefuroxime was the initial empiric antimicrobial agent given for 66/76 of the patients and treatment outcome was favorable for 20/22 patients who received cefuroxime at least up to day 5. All isolates were susceptible to benzylpenicillin and cefuroxime interpreted by EUCAST breakpoints for Aerococci and PK-PD breakpoints, respectively. MIC determinations gave variable results for ceftriaxone, 2.1-2.9\% of the isolates were resistant. To conclude, it seems that the number of bacteraemic Aerococcus urinae cases is increasing at Helsinki metropolitan area, Finland, reflecting the growing blood culture sampling. Clinical A. urinae isolates were susceptible to cefuroxime in vitro. Treatment data indicate that empirical cefuroxime started for possibly urinary tract -derived community-acquired bacteraemia covers $A$. urinae.
\end{abstract}

Keywords Aerococcus urinae $\cdot$ Bacteraemic infections $\cdot$ Susceptibility testing $\cdot$ Benzylpenicillin $\cdot$ Cefuroxime $\cdot$ Ceftriaxone

Reetta Sihvonen

reetta.sihvonen@hus.fi

1 Department of Clinical Microbiology, HUSLAB, University of Helsinki and Helsinki University Hospital, HUS Diagnostic Center, Haartmaninkatu 3, N00029 HUS, Helsinki, Finland

2 Faculty of Biological and Environmental Sciences, Molecular and Integrative Biosciences Research Programme, University of Helsinki, Helsinki, Finland

3 Department of Infectious Diseases, Inflammation Center, Helsinki University Central Hospital, Helsinki, Finland

4 Clinical Microbiology, Turku University Hospital and University of Turku, Turku, Finland

\section{Introduction}

Aerococcus urinae, a Gram-positive coccus, causes urinary tract infections and invasive infections like bacteraemia and infective endocarditis, especially among the elderly [1-6]. In addition to old age, predisposing factors suggested to be associated with $A$. urinae bacteraemia are male gender and underlying urological conditions including catheterization $[1,2]$. As a newcomer and rare pathogen, A. urinae seems to be an unfamiliar microorganism to most clinicians and the treatment approaches are variable $[1,7]$. In Europe, bacteraemic A. urinae infections has been characterized comprehensively only in Sweden [2, 7]. Aging in the human population generates global challenges [8] and the resulting multimorbidity may raise the significance of the emerging pathogens like A. urinae in the future. A proper managing of infections caused by $A$. 
urinae is important also from the antibiotic stewardship perspective. Aerococci are catalase negative and resemble $\alpha$-hemolytic streptococci when growing on blood agar plate but in Gram staining cells appear in clusters like staphylococci [9]. MALDI-TOF mass spectrometry (MS) techniques have substantially improved correct identification of aerococci in clinical microbiology laboratories and contributed to its emergence as a "novel" agent of urinary tract derived infections $[2,10]$.

Antimicrobial susceptibility breakpoints or interpretive criteria are needed to define clinical susceptibility or resistance of a microbe to a certain antimicrobial. In vitro susceptibility of $A$. urinae has been studied to some extent but there is variation in the used methods and result interpretations $[11,12]$. The European Committee on Antimicrobial Susceptibility Testing (EUCAST) recently released clinical breakpoints for Aerococcus urinae against certain penicillins, fluoroquinolones, meropenem, vancomycin, nitrofurantoin, and rifampicin [13]. In Finland, the empiric starting therapy for community-acquired bacteraemias is the secondgeneration cephalosporin cefuroxime. However, EUCAST standard lacks the clinical breakpoints for aerococci and cephalosporins. The aim of the study was to assess the incidence of bacteraemic infections caused by $A$. urinae in Helsinki metropolitan area during the years 2013-2018 and to characterize these cases by reviewing the medical charts retrospectively. We aimed to estimate the treatment outcome of cefuroxime in managing these infections. Three different susceptibility testing methods, i.e., disk diffusion (EUCAST methodology), gradient test (Etest), and broth microdilution (Sensititre), were compared to study in vitro susceptibilities for benzylpenicillin, cefuroxime, and ceftriaxone of a set of clinical A. urinae isolates.

\section{Materials and methods}

\section{Isolates for susceptibility testing}

Our laboratory, HUSLAB (Hospital District of Helsinki and Uusimaa Laboratory Services), serves the Helsinki metropolitan area of approximately 1.7 million inhabitants and the specimen catchment area has remained constant during the study period. For susceptibility testing, available stored $\left(-70{ }^{\circ} \mathrm{C}\right)$ clinical blood culture Aerococcus urinae isolates from the years 2013-2018 $(n=81)$ were retrospectively searched from the laboratory database using the WHONET software and the urine culture isolates $(n=60)$ were collected and stored for this study during 2 months in November-December 2017. Isolates were subcultured on horse blood agar, grown in $5 \% \mathrm{CO}_{2}$ at $35 \pm 1{ }^{\circ} \mathrm{C}$ for $16-20 \mathrm{~h}$ before species identification using MALDI-TOF MS (VITEK MS MALDI-TOF, bioMérieux, France).

\section{Antimicrobial susceptibility testing}

Susceptibility testing of A. urinae isolates for benzylpenicillin, cefuroxime, and ceftriaxone was performed in parallel by three different methods; disk diffusion (Oxoid, Cambridge, UK), gradient tests (Etest, BioMérieux SA, Marcy I'Etoile, France), and broth microdilution (Sensititre, Thermo Fisher Scientific, Pittsburgh, PA.). Disk diffusion tests were performed in duplicate according to EUCAST methodology for fastidious organisms. A McFarland 0.5 inoculum measured by Densimat (Biomerieux, SA, Marcy I'Etoile, France) was used on MH-F plates and incubated at $35 \pm 1{ }^{\circ} \mathrm{C}$ in $5 \% \mathrm{CO}_{2}$ for $16-20 \mathrm{~h}$. The concentrations of the antibiotic disks were 1 unit for benzylpenicillin, $30 \mu \mathrm{g}$ for cefuroxime, and $30 \mu \mathrm{g}$ for ceftriaxone. Gradient tests were performed according to the manufacturer's instructions in duplicates. Etest gradients used were $0.002-32 \mathrm{mg} / \mathrm{L}$ for benzylpenicillin, $0.016-256 \mathrm{mg} / \mathrm{L}$ for cefuroxime, and $0.016-256 \mathrm{mg} / \mathrm{L}$ for ceftriaxone. If necessary, isolates were re-incubated up to $40-44 \mathrm{~h}$. For broth microdilution, Sensititre Streptococcus species STP6F plate was used. The ranges of penicillin, cefuroxime, and ceftriaxone were $0.03-4 \mathrm{mg} / \mathrm{L}$, $0.5-4 \mathrm{mg} / \mathrm{L}$, and $0.12-2 \mathrm{mg} / \mathrm{L}$, respectively. One hundred microliters of $0.5 \mathrm{McFarland}$ suspension was transferred to $11 \mathrm{~mL}$ of Mueller-Hinton broth with lysed horse blood (Thermo Fisher Scientific) and inoculated onto the STP6F plates by Sensititre AIM ${ }^{\mathrm{TM}}$ Automated Inoculation Delivery System and incubated for $24 \mathrm{~h}$ at $35^{\circ} \mathrm{C}$. Minimal inhibitory concentration (MIC) values were read using Sensititre Vizion $^{\mathrm{TM}}$ Digital MIC Viewing System and the Sensititre SWIN Software with semi-automated read options. Streptococcus pneumoniae ATCC 49619 was used as the quality control strain for all examined antimicrobial agents.

\section{Clinical characterization of bacteraemic patients}

Medical charts of 77 patients were available for the study and the underlying diseases, symptoms (at admission and during the clinical course of the disease), signs of sepsis (e.g., low blood pressure, higher respiratory rate, altered mental status), and laboratory and imaging findings were evaluated. The antimicrobial treatment, i.e., agents used and length in days throughout the infection episode, was recorded. Evaluations for metastatic infectious lesions and 30-day mortality were recorded. A patient was considered to respond to the antimicrobial therapy if she/he was feverless and if CRP value had decreased by $\geq 10 \%$ within days 5-9. Helsinki University Hospital (HUS) and the City of Helsinki approved this study. 


\section{Statistical analysis}

Statistical analysis was performed by SPSS Statistics ver. 25.0 (IBM Co., Armonk, NY, USA). Chi-square test was used to study the incidence between the study years. In general, Independent sample $t$-test was used for constant variables and Mann-Whitney U-test for categorical variables and for small sample sizes. Wilcoxon signed-rank test and Pearson correlation were used to compare gradient test and broth microdilution. Pearson correlation was used to evaluate the association between benzylpenicillin and cefuroxime susceptibility results. A $p$-value of $<0.05$ was considered statistically significant.

\section{Results}

\section{Aerococcus urinae bacteraemic patients}

\section{Incidence and microbiological findings}

During the 6-year study period (years 2013-2018), altogether, 94 patients were diagnosed with bacteraemia caused by $A$. urinae (identified by MALDI-TOF MS) corresponding to incidence of 9.2 cases per 1,000,000 inhabitants per year. Figure 1 represents bacteraemic $A$. urinae cases distributed throughout the study years and the trend seems to be increasing $(p=0.01)$. In the same period, the total amount of taken blood culture samples has also increased steadily from 100,801 blood culture bottles drawn in 2013 to 155,092 bottles drawn in 2018.

Electronic medical charts of 77 (81.9\%) patients were accessible and the results given below represent them. Besides A. urinae, 27.3\% (21/77) of the patients had one or more additional bacterial species in their blood culture samples. Urine culture showed A. urinae in 7.8\% (6/77), mixed growth in $26.0 \%$ (20/77), and other uropathogens in $14.3 \%$ (11/77) of the cases. Blood and urine culture findings are described in Table 1.

\section{Patient characteristics and symptoms}

Table 2 defines the clinical characteristics of the bacteraemic patients. They were mainly elderly and male. All patients suffered from one or multiple underlying conditions. Vast majority had cardiovascular disease and three-fourths some underlying urologic or nephrologic disorder. Fever and urinary tract symptoms were common. One-fifth of the patients died within 30 days and they were significantly older compared to the patients who survived ( 86 years versus 78 years, respectively, $p=0.018$ ). Neither group had polymicrobial bacteremia more often $(p=0.147)$.

\section{Clinical findings during disease}

Table 3 shows the initial clinical presentations and the follow-up data. Most patients presented with fever and increased inflammatory markers at the onset of infection. Both CRP and total white cell counts decreased by days 5-9 and only four patients still had fever. Notably, the patients who died had significantly higher CRP within days 5-9 compared to the patients who survived $(96 \mathrm{mg} / \mathrm{L}$ versus $43 \mathrm{mg} / \mathrm{L}$, respectively, $p=0.003$ ).

The following radiological examinations were performed: abdominal/urinary tract computed tomography (CT) for $13 / 77$ (16.9\%) of the patients, body CT scan for 7/77 (9.1\%), chest CT scan for 2/77 (2.6), abdominal ultrasound for $17 / 77$ (22.1\%), urinary tract ultrasound for $6 / 77$ (7.8\%), and transthoracic/transesophageal echocardiography for 17/77 (22.1\%). Metastatic infectious lesions were rare.
Fig. 1 Bacteraemic A. urinae cases per study years $2013-$ $2018(n=94)$

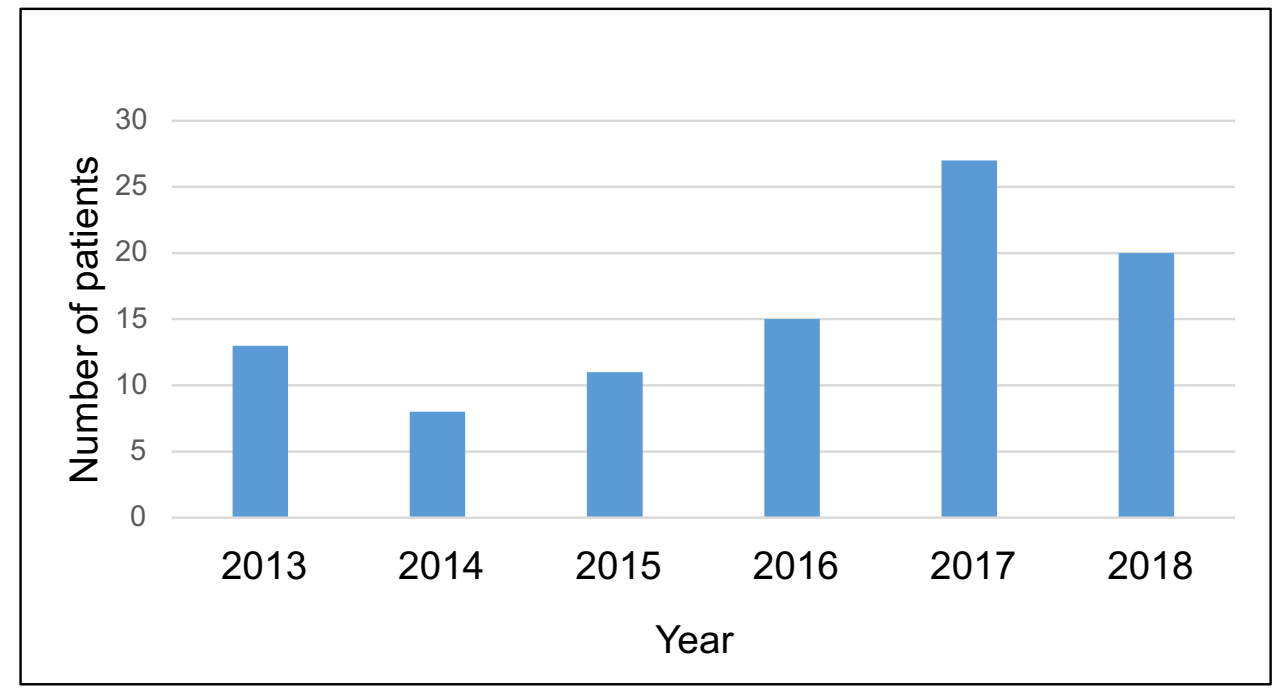


Table1 Blood and urine culture findings of the bacteraemic patients

\begin{tabular}{|c|c|c|}
\hline Culture finding & Number $(\%)$ & Bacterial species \\
\hline \multicolumn{3}{|l|}{ From blood } \\
\hline Pure culture & $56 / 77(72.7)$ & Aerococcus urinae \\
\hline A. urinae and one additional species & $18 / 77(23.4)$ & $\begin{array}{l}\text { Enterococcus faeclis } n=4 \\
\text { Proteus mirabilis }{ }^{a} n=3 \\
\text { Escherichia coli, Staphylococcus aureus } n=2 \\
\text { Klebsiella pneumoniae }{ }^{b} \text {, Streptococcus anginosus group, } \\
\text { Streptococcus agalactiae, lactobacillus, Actinotignum schaalii, } \\
\text { Bacteroides fragilis group, Clostridium perfringens } n=1\end{array}$ \\
\hline A. urinae and two additional species & 3/77 (3.9) & $\begin{array}{l}\text { E. faecalis and Staphylococcus epidermidis } \\
\text { Peptinophilus asaccharolyticus and coagulase negative staphylococcus } \\
\text { P. asaccharolyticus and A. schaalii }\end{array}$ \\
\hline \multicolumn{3}{|l|}{ From urine } \\
\hline Pure culture & $6 / 77(7.8)$ & A. urinae \\
\hline Other uropathogen & $11 / 77(14.3)$ & $\begin{array}{l}\text { K. pneumoniae }{ }^{b}, P . \text { mirabilis }^{a}, \text { E. faecium } n=3 \\
\text { E. coli, Klebsiella oxytoca, Enterobacter cloacae, S. agalactiae } n=1\end{array}$ \\
\hline Mixed growth ${ }^{c}$ & 20/77 (26.0) & - \\
\hline Negative (no growth) & $6 / 77(7.8)$ & - \\
\hline Other status $^{\mathrm{d}}$ & $34 / 77(44.2)$ & - \\
\hline
\end{tabular}

${ }^{a}$ Both in blood and urine in two cases

${ }^{b}$ Both in blood and urine in one case

${ }^{c}>2$ species without any predominant primary urinary tract pathogen

${ }^{\mathrm{d}}$ Urine culture not taken or taken after antimicrobial started

Two patients (2.6\%) had diagnosed endocarditis, one patient (1.3\%) suffered from spondylodiscitis, and three patients (3.9\%) had abscesses (1 kidney, 1 paravertebral abscess, and 1 subcutaneal abscess in the lower abdominal wall). In addition, one patient had $S$. aureus abscess in an ankle simultaneously with the A. urinae bacteremia. Most patients did not undergo any radiological examinations to search for metastatic infectious lesions.

\section{Antimicrobial therapy}

Table 4 summarizes the used antimicrobial agents. One patient apparently did not receive any antimicrobial treatment (and survived the infection). In initial therapy, eight different antimicrobials or their combinations were used: cefuroxime, cefuroxime combined with tobramycin, cefuroxime combined with metronidazole, ceftriaxone, amoxicillin, cloxacillin, piperacillin-tazobactam, or ciprofloxacin. Cefuroxime was the initial empiric antimicrobial given for majority of the patients $(86.8 \%, 66 / 76)$ with a typical dose of $1.5 \mathrm{~g}$ three times daily intravenously. Cefuroxime was equally used as the initial drug among patients who died $(82.4 \%, 14 / 17)$ and survived $(88.1 \%, 52 / 59)(p=0.537)$. The mean length of the initial cefuroxime treatment was 4 days (range 1-11). On day 5 after hospitalization, cefuroxime was given for $28.9 \%$ (22/76) of the patients, of which majority $(90.9 \%, 20 / 22)$ survived. There were no differences in cefuroxime MIC values between dead and survivors $(p=0.339$ for gradient tests; $p=0.100$ for broth microdilution). Antimicrobial de-escalation from the first empirical choice to benzylpenicillin, ampicillin, or amoxicillin (with or without clavulanic acid) during days $1-5$ was introduced among $30.3 \%$ (23/76) of the cases. In the remaining cases $(69.7 \%, 53 / 76)$, the choice of the second, third, and further antimicrobial agents seemed rather random.

\section{Antimicrobial susceptibilities}

Figure 2 shows that MICs of blood and urine isolates distributed quite equally, and all studied isolates were susceptible (S) to benzylpenicillin and cefuroxime. According to gradient tests, nearly all isolates $(97.9 \%, 137 / 140)$ were susceptible to ceftriaxone but $5.7 \%$ (8/140) were susceptible with increased exposure (I). The remaining 2.1\% (3/140) of the isolates were resistant $(\mathrm{R})$ to ceftriaxone. Broth microdilution gave rather similar percentages for ceftriaxone: $97.1 \%$ $(135 / 139)$ of the isolates were S, $10.1 \%$ (14/139) were I, and $2.9 \%(4 / 139)$ were R. Benzylpenicillin MICs were interpreted according to EUCAST $A$. urinae breakpoints $(\mathrm{S} \leq 0.125, \mathrm{R}>0.125)$, while EUCAST PK-PD breakpoints were used to interpret cefuroxime $(S \leq 4, R>8)$ and ceftriaxone $(\mathrm{S} \leq 1, \mathrm{R}>2)$ MICs. 
Table 2 Characteristics of the bacteraemic patients including demographics, underlying diseases, the initial signs and symptoms, and other features

\begin{tabular}{|c|c|}
\hline Characteristics & No. of patients $(n=77)$ \\
\hline \multicolumn{2}{|l|}{ Demographics } \\
\hline Mean age in years (range) & $80(47-98)$ \\
\hline Male gender & $61(79.2 \%)$ \\
\hline \multicolumn{2}{|l|}{ Underlying disease $^{\mathrm{a}}$} \\
\hline Patients with cardiovascular diseases & $65(84.4 \%)$ \\
\hline Hypertension & $48(62.3 \%)$ \\
\hline Atrial fibrillation & $26(33.8 \%)$ \\
\hline Ischemic heart disease & $17(22.1 \%)$ \\
\hline $\begin{array}{l}\text { Patients with urologic or nephrologic } \\
\text { disorder }\end{array}$ & $57(74.0 \%)$ \\
\hline Benign prostatic hypertrophy & $25(41.0 \%)$ \\
\hline Prostatic cancer & $13(21.3 \%)$ \\
\hline Indwelling urinary catheter & $17(22.1 \%)$ \\
\hline $\begin{array}{l}\text { Urinary tract strictures or stone } \\
\text { formation }\end{array}$ & $18(23.4 \%)$ \\
\hline Patients with neurologic disorder & $51(66.2 \%)$ \\
\hline Memory disorder & $28(36.4 \%)$ \\
\hline Cerebrovascular disorder & $23(29.9 \%)$ \\
\hline $\begin{array}{l}\text { Patients with other malignancy (current } \\
\text { or past) }\end{array}$ & $13(16.9 \%)$ \\
\hline Patients with diabetes & $16(20.8 \%)$ \\
\hline \multicolumn{2}{|l|}{ Signs and symptoms (at hospital arrival) ${ }^{a}$} \\
\hline Fever $\left(\geq 38^{\circ} \mathrm{C}\right)^{\mathrm{c}}$ & $63(81.8 \%)$ \\
\hline Urinary tract symptoms & $45(58.4 \%)$ \\
\hline Functional decline & $31(40.3 \%)$ \\
\hline Cognitive decline & $15(19.5 \%)$ \\
\hline Gastrointestinal symptoms & $21(27.3 \%)$ \\
\hline \multicolumn{2}{|l|}{ Other symptoms } \\
\hline Cardiac symptoms & $11(14.3 \%)$ \\
\hline Articular symptoms & $5(6.5 \%)$ \\
\hline \multicolumn{2}{|l|}{ Other } \\
\hline 30-day mortality & $17(22.1 \%)$ \\
\hline $\begin{array}{l}\text { Mean duration of hospitalization in days } \\
\text { (range) }\end{array}$ & $20(0-126)$ \\
\hline Intensive care unit (ICU) admission & $7(9.1 \%)$ \\
\hline Mean duration of ICU in days (range) & $6(1-15)$ \\
\hline Institutional care & $21(27.3 \%)$ \\
\hline Penicillin allergy & $3(4.1 \%)^{d}$ \\
\hline
\end{tabular}

${ }^{a}$ No significant difference between died and survived patients (data not shown)

${ }^{\mathrm{b}}$ Percentage of men $(n=61)$

${ }^{\mathrm{c}}$ Mentioned in the preliminary report or measured during hospitalization

${ }^{\mathrm{d}}$ Number of patients $n=73$

Table 5 describes the susceptibility results performed by disk diffusion method and compares the results obtained from MIC determinations. MIC values performed by gradient test and broth microdilution correlated statistically significantly among all tested antimicrobial agents. However, broth microdilution gave on average higher MIC values, even when gradient test values were converted to correspond to broth microdilution values (analyzed by Wilcoxon signed-rank test, data not shown). There was also discrepancy among ceftriaxone MIC interpretations. Two out of 139 isolates (1.4\%) were $\mathrm{R}$ by broth microdilution but $\mathrm{S}$ or I by gradient test (very major errors). Vice versa, 1/139 isolate $(0.7 \%)$ was $\mathrm{R}$ by gradient test but $\mathrm{S}$ by broth microdilution (a major error). According to disk diffusion, all isolates were susceptible to benzylpenicillin $(\mathrm{S} \geq 21 \mathrm{~mm})$ interpreted using EUCAST A. urinae breakpoints. Penicillin and cefuroxime zone diameters correlated significantly (Pearson correlation $0.924, p<0.001)$.

\section{Discussion}

We found an increasing number of bacteraemic Aerococcus urinae cases at Helsinki Metropolitan area, Finland. This may reflect the increase in total amount of blood culture samples drawn. Nevertheless, this observation is worth noticing as aging in human population may rise the significance of A. urinae as a causative organism in blood stream infections among elderly. Further surveillance is needed to follow up the incidence.

Consistently with previous studies [1,2], we found that bacteraemic patients with $A$. urinae were mainly elderly men with several comorbidities. A. urinae was found only in a small fraction of the urine samples although urogenital tract was the probable infection focus on many cases, as in previously published data [7]. Mixed flora reported in urine cultures may have covered possible underlying A. urinae growth, which calls for the importance of proper urine sampling techniques and experience. However, also nearly onethird of bacteremic cases showed polymicrobial blood culture findings. It may be possible that $A$. urinae in blood culture originates from another source than the urinary tract, e.g., from the upper respiratory tract or from the gastro-intestinal system. In surprisingly many cases, urine sample was taken only after antimicrobial therapy had been initiated or not at all. Metastatic infectious lesions were found only seldom but notably deep focuses were not systemically searched for in all patients due to their poor condition or old age.

In our cohort, the $22 \%$ mortality rate was somewhat higher than reported previously $[1,2,4,7,14]$. In other studies, 30-day mortality has ranged between 6 and 17\%. We found that patients who died were on average older compared to survivors. Patients responded to the given antimicrobial treatment mostly well based on declined fever and CRP. However, CRP declined significantly slower for patients who died. Mortality was not enhanced by polymicrobial finding 
Table 3 Clinical and laboratory findings of the bacteraemic patients. Initial finding (Day $0)$ refers to the first parameter measured after hospitalization

\begin{tabular}{llll}
\hline Finding & Initial = day 0 & Days 0-4 & Days 5-9 \\
\hline Mean temperature ${ }^{\circ} \mathrm{C}$ (range) & $38.0(33.2-40.0)$ & - & - \\
Fever $\geq 38^{\circ} \mathrm{C}$ & $45 / 77(58.4 \%)$ & - & $4 / 73(5.5 \%)$ \\
Mean CRP, mg/L (range) & $94(<3-416)$ & $185(25-466)$ & $53(<3-278)^{\mathrm{a}}$ \\
Mean leucocyte, E9/L (range) & $12.2(1.3-40.2)$ & $17.3(2.0-40.8)$ & $9.4(4.5-22.4)^{\mathrm{a}}$ \\
Kidney insufficiency GFR $^{\mathrm{b}}<60^{\mathrm{c}}$ & $39 / 75(52.0 \%)$ & - & - \\
${\text { Hypotension }<100 \mathrm{mmHg}^{\mathrm{a}} \text { systolic }}^{\text {Response to therapy }}$ & $11 / 76(14.5 \%)$ & - & - \\
\hline
\end{tabular}

${ }^{\text {a }}$ Statistically significantly higher in patients who died within 30 days

${ }^{\mathrm{b}} G F R$, glomerular filtration rate

${ }^{c}$ Women had kidney insufficiency more often than men (86\% versus $44 \%$, respectively, $p=0.005$ )

${ }^{\mathrm{d}}$ No fever and CRP decreased $\geq 10 \%$

${ }^{\mathrm{e}} \mathrm{Complete}$ data (CRP value or temperature ${ }^{\circ} \mathrm{C}$ ) was missing from 12 patients

Table 4 Antimicrobial treatment of bacteraemic patients $(n=77)$

\begin{tabular}{|c|c|c|c|c|c|}
\hline Antimicrobial agent & Initial agent $(n=76)$ & 2 nd agent $(n=70)$ & 3rd agent $(n=45)$ & 4 th agent $(n=21)$ & 5th agent $(n=6)$ \\
\hline Cefuroxime & $66(86.8 \%)$ & $1(1.4 \%)$ & $5(11.1 \%)$ & - & $1(16.7 \%)$ \\
\hline Cefuroxime + other agenta & $3(3.9 \%)$ & $5(7.1 \%)$ & $1(2.2 \%)$ & $1(4.8 \%)$ & - \\
\hline Ceftriaxone & $2(2.6 \%)$ & $4(5.7 \%)$ & $4(8.9 \%)$ & $4(14.3 \%) b$ & - \\
\hline Cefalexin & - & $3(4.3 \%)$ & $1(2.2 \%) \mathrm{c}$ & $1(4.8 \%)$ & - \\
\hline Penicillins & $2(2.6 \%)$ & $34(48.6 \%)$ & $22(48.9 \%)$ & $9(42.9 \%)$ & $3(50 \%)$ \\
\hline Benzylpenicillin & - & $11(15.7 \%)$ & $4(8.9 \%)$ & $4(19.0 \%)^{\mathrm{d}}$ & - \\
\hline Phenoxymethylpenicillin & - & - & $4(8.9 \%)$ & $1(4.8 \%)$ & $2(33.3 \%)^{\mathrm{d}}$ \\
\hline Ampicillin & - & $7(10.0 \%)^{\mathrm{d}}$ & $1(2.2 \%)$ & - & - \\
\hline Amoxicillin & $1(1.3 \%)$ & $7(10.0 \%)$ & $12(26.7 \%)^{\mathrm{c}}$ & $2(9.5 \%)$ & $1(16.7 \%)$ \\
\hline $\begin{array}{l}\text { Amoxicillin with clavulanic } \\
\text { acid }\end{array}$ & - & $5(7.1 \%)$ & $1(2.2 \%)$ & $2(9.5 \%)$ & - \\
\hline Cloxacillin & $1(1.3 \%)$ & $4(5.7 \%)^{\mathrm{e}}$ & - & - & \\
\hline Broad-spectrum $^{f}$ & $4(5.3 \%)$ & $11(15.7 \%)$ & $5(11.1 \%)^{\mathrm{d}}$ & $2(9.5 \%)$ & $2(33.3 \%)$ \\
\hline Other ${ }^{\mathrm{g}}$ & $1(1.3 \%)$ & $12(17.1 \%)$ & $7(15.6 \%)^{\mathrm{h}}$ & $4(19.0 \%)$ & - \\
\hline
\end{tabular}

${ }^{\mathrm{a} O t h e r}$ agent $=$ clindamycin, metronidazole, tobramycin, moxifloxacin, and vancomycin

${ }^{\mathrm{b}}$ Three cases combined with (1) clindamycin (2) piperacillin-tazobactam and (3) linezolid

${ }^{\mathrm{c}}$ One case combined with metronidazole

${ }^{\mathrm{d}}$ One case combined with levofloxacin

${ }^{\mathrm{e}}$ One case combined with vancomycin

${ }^{\mathrm{f}}$ Imipenem, meropenem, and piperacillin-tazobactam

${ }^{g}$ Ciprofloxacin, clindamycin, gentamicin, levofloxacin, nitrofurantoin, sulfa-trimethoprim, and vancomycin

${ }^{\mathrm{h}}$ In one case levofloxacin combined with vancomycin

in blood culture. As far as we know, cefuroxime treatment for bacteraemic A. urinae infections has not been reported before. Cefuroxime was the first empiric antimicrobial choice in most of the cases and based on our data, we did not find evidence against its use. Rasmussen [9] states in his review article that the use of cephalosporins in A. urinae infections lack rationale due to worse pharmacodynamics apparently comparing to penicillin. Nevertheless, we suppose that empirical cefuroxime treatment for a suspected urinary tract-derived community-acquired bacteraemia also covers A. urinae, a rare causative agent in bacteraemic infections. De-escalation to benzylpenicillin, ampicillin, or amoxicillin should be performed when A. urinae is recognized by name in blood culture report and penicillin allergy or mixed infection is not a concern.

All tested A. urinae isolates were susceptible to cefuroxime (MIC $\leq 4 \mathrm{mg} / \mathrm{L}$, using EUCAST PK-PD breakpoints) and clinical response was not inferior compared to other agents used in the studied patient cohort. In accordance with the previous findings [7, 15], all isolates were 
a

b

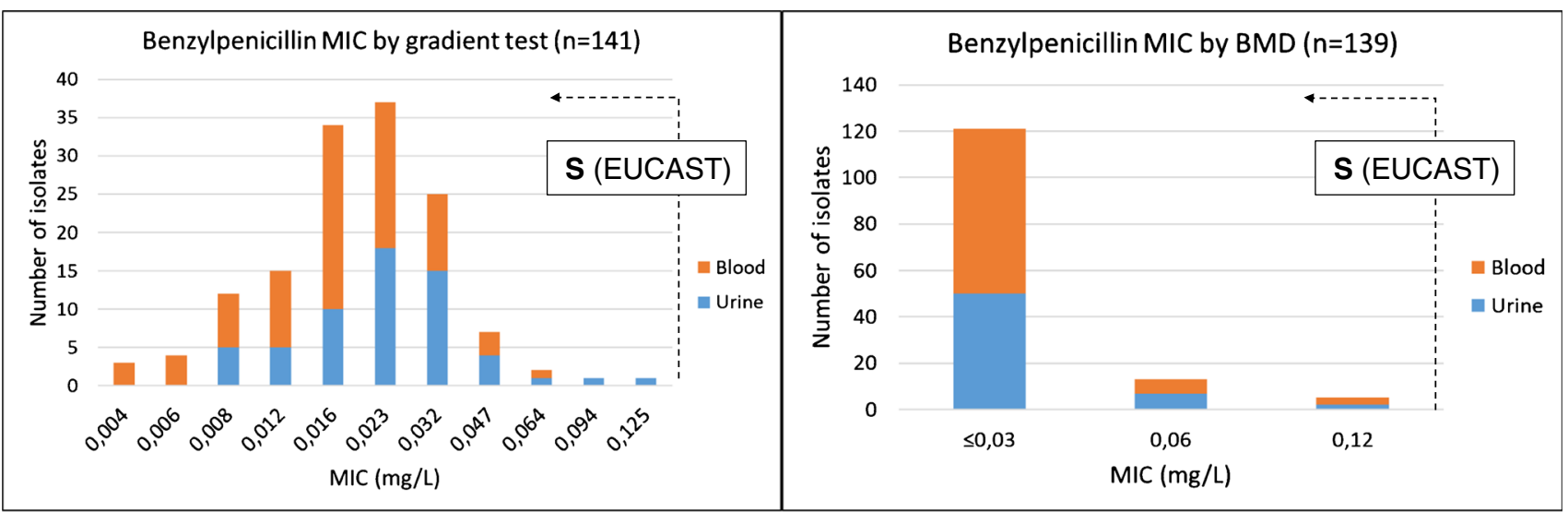

C

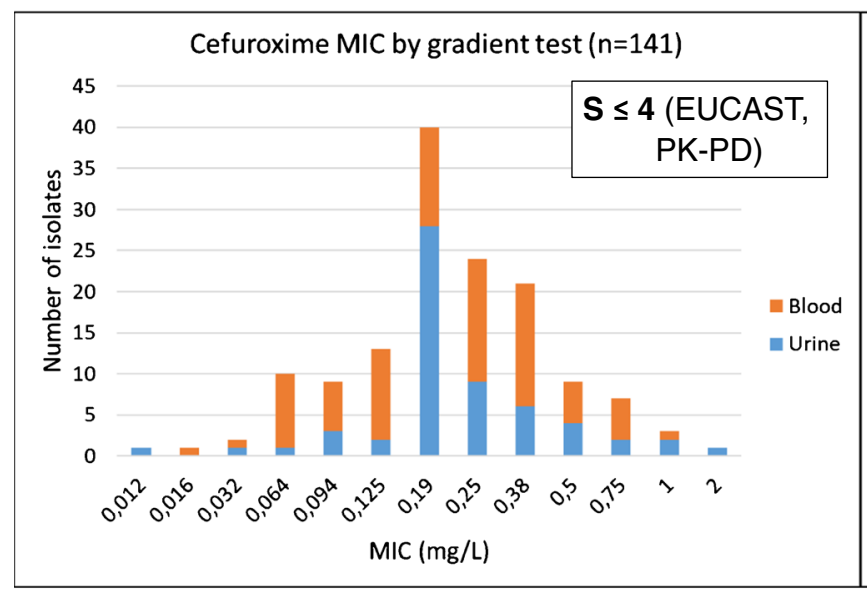

e

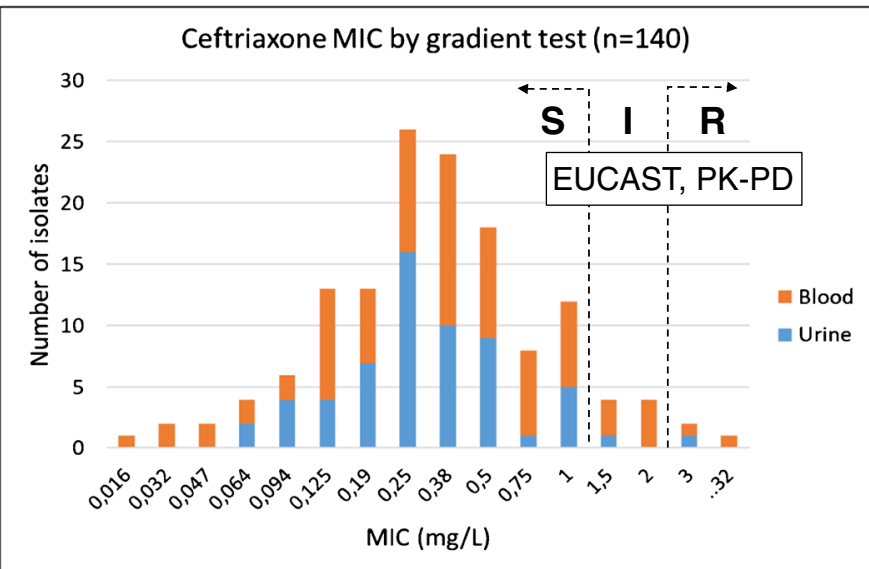

Fig. 2 Minimal inhibitory concentrations (MICs) against A. urinae isolates performed by gradient diffusion test and broth microdilution method (BMD). a Benzylpenicillin MICs by gradient diffusion. b Benzylpenicillin MICs by BMD. c Cefuroxime MICs by gradient diffusion. $\mathbf{d}$ Cefuroxime MICs by BMD. e Ceftriaxone MICs by gradi- d

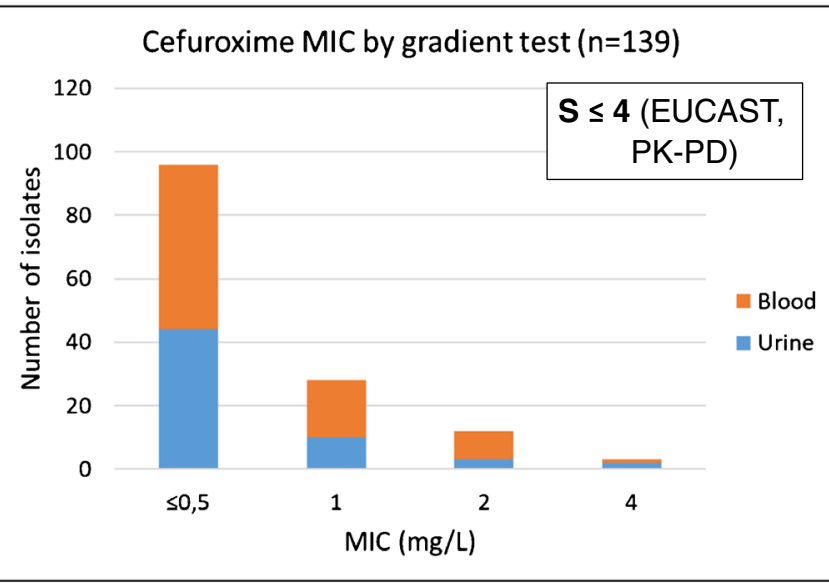

f

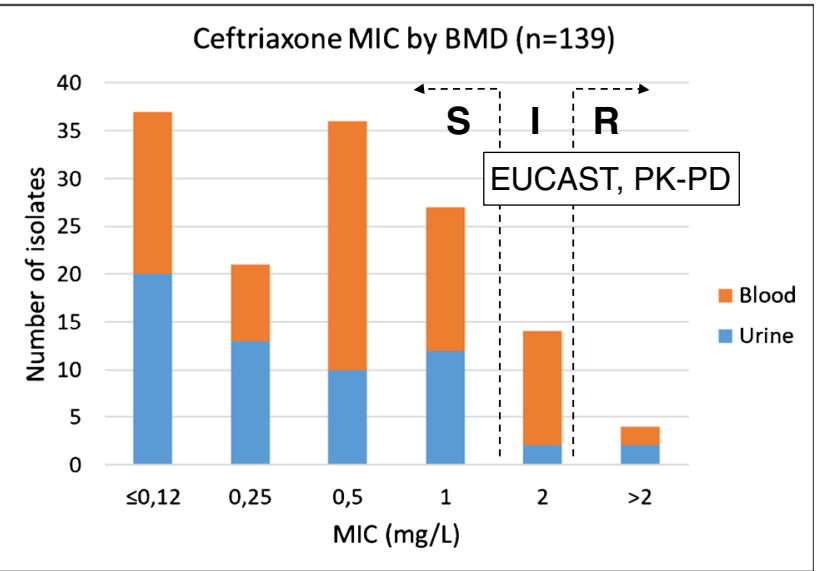

ent diffusion. f Ceftriaxone MICs by BMD. For penicillin, EUCAST breakpoints for Aerococci were used to interpret the results. For cefuroxime and ceftriaxone, EUCAST PK-PD breakpoints were used. $S$, susceptible; $I$, susceptible; increased exposure; $R$, resistant; $P K$ $P D$, pharmacokinetic-pharmacodynamic 
Table 5 The effects of the antimicrobial agents benzylpenicillin, cefuroxime and ceftriaxone against 139-141 Aerococcus urinae isolates. Zone diameters (mm), MIC50, MIC90, and MIC ranges are

\begin{tabular}{|c|c|c|c|c|c|c|c|c|c|c|}
\hline \multirow[t]{3}{*}{ Antimicrobial agent } & \multirow{3}{*}{$\begin{array}{l}\text { Disk } \\
\text { diffusion } \\
\mathrm{mm}\end{array}$} & \multicolumn{4}{|c|}{ Gradient test } & \multicolumn{4}{|c|}{ Broth microdilution (BMD) } & \multirow{3}{*}{$\begin{array}{l}\text { Gradient vs. BMD } \\
\text { Pearson correlation }\end{array}$} \\
\hline & & \multicolumn{4}{|c|}{ MIC (mg/L) } & \multicolumn{4}{|c|}{$\mathrm{MIC}(\mathrm{mg} / \mathrm{L})$} & \\
\hline & & $\mathrm{MIC}_{50}{ }^{\mathrm{a}}$ & $\mathrm{MIC}_{90}{ }^{\mathrm{b}}$ & Range & $\mathrm{S} / \mathrm{I} / \mathrm{R}^{\mathrm{c}}$ & $\mathrm{MIC}_{50}{ }^{\mathrm{a}}$ & $\mathrm{MIC}_{90}{ }^{\mathrm{b}}$ & Range & $\mathrm{S} / \mathrm{I} / \mathrm{R}^{\mathrm{c}}$ & \\
\hline Benzylpenicillin & $26-47$ & 0.023 & 0.032 & $0.004-0.125$ & $141 / 0 / 0$ & $\leq 0.03$ & 0.06 & $\leq 0.03-0.12$ & $139 / 0 / 0$ & $0.428, p<0.001$ \\
\hline Cefuroxime & $24-47$ & 0.19 & 0.5 & $0.012-2$ & $141 / 0 / 0$ & 0.5 & 2 & $0.5-4.0$ & $139 / 0 / 0$ & $0.622, p<0.001$ \\
\hline Ceftriaxone & $23-47$ & 0.38 & 1.0 & $0.016-32$ & $129 / 8 / 3$ & 0.5 & 2 & $0.12->2$ & $121 / 14 / 4$ & $0.579, p<0.001$ \\
\hline
\end{tabular}

${ }^{\mathrm{a}} \mathrm{MIC}$ value at which $\geq 50 \%$ of the isolates are inhibited

${ }^{\mathrm{b}} \mathrm{MIC}$ value at which $\geq 90 \%$ of the isolates are inhibited

${ }^{\mathrm{c}} S$, susceptible; $I$, susceptible, increased exposure; $R$, resistant

susceptible to benzylpenicillin. Benzylpenicillin and cefuroxime zone diameters correlated well, suggesting that susceptibility to cefuroxime could be interpreted from benzylpenicillin zone millimeters. On the contrary, the ceftriaxone MIC distribution showed that $2.1-2.9 \%$ of the isolates were resistant to ceftriaxone (MIC $>2 \mathrm{mg} / \mathrm{L}$ ). In our study, ceftriaxone was used only for few patients, so we were not able to analyze the treatment outcome of the agent. Ceftriaxone susceptibility may need separate testing, and not interpretation from benzylpenicillin susceptibility. Ceftriaxone resistance among $A$. urinae has been described previously $[5,11,16,17]$ but its clinical relevance has not yet been resolved. The proportion of resistant isolates depends on the interpretation criteria. When EUCAST breakpoints for viridans group streptococci $(\leq 0.5 \mathrm{mg} / \mathrm{L})$ were used, $76 \%$ of the studied A. urinae isolates were considered susceptible [9]. In our material, $107 / 139(77 \%)$ of the studied isolates had ceftriaxone MIC value $\leq 0.5 \mathrm{mg} / \mathrm{L}$. Finnish A. urinae isolates resemble those studied in other countries when concerning benzylpenicillin and ceftriaxone in vitro susceptibility.

Carkaci and co-workers [15] reported parallel susceptibility testing on A. urinae isolates by gradient test and broth microdilution and they found that penicillin and cefotaxime MICs were in accordance within one dilution. In our study, broth microdilution gave somewhat higher MIC values compared to gradient test for all studied antimicrobials. Previous studies have reported inconsistent MIC values obtained by Etest versus broth microdilution, e.g., for fluoroquinolones [18] and for tetracycline and erythromycin [19]. Clinically most serious implications result when true-resistant isolate is categorized as susceptible leading likely to therapeutic failure [20]. Ceftriaxone MIC interpretations from our data resulted in two very major errors and one major error. Cantón and colleagues [21] determined Staphylococcus aureus ceftaroline MICs and found that isolates were more often ceftaroline resistant by broth microdilution as compared to Etest. In addition, discrepancy observed with $A$. described. The correlation and the number of susceptibility interpretations (S/I/R) between MIC methods are shown 
of bacteraemic cases were polymicrobial and cefuroxime covered most of these findings. In bacteraemic cases caused by $A$. urinae alone, definitive penicillin therapy should be considered. Our study complements the understanding about bacteraemic infections caused by Aerococcus urinae and introduces a set of cefuroxime in vitro susceptibility data for this species.

Acknowledgements We want to thank Ida Hattunen, Ida Nissinen, and Julia Olin from Metropolia University of Applied Sciences and Lotta Maamies, Tuulimarja Törnberg, and Sirpa Vainonen from HUSLAB for excellent contribution to susceptibility testing (disk diffusion and gradient test).

Author contribution Pätäri-Sampo A designed and supervised the study, applied for the research permission at HUCS, and contributed to writing of the manuscript. Sihvonen R designed the study and applied for the permissions from HUCS and the City of Helsinki. Sihvonen organized the susceptibility testing at HUSLAB, interpreted disk diffusion and gradient test results, made all figures and tables, was mainly responsible for the statistical analyses, and was primarily responsible for the writing of the manuscript. Turunen M, Lehtola L, and Pakarinen $\mathrm{L}$ investigated the medical records, helped to interpret the clinical patient data, and reviewed the manuscript. Turunen $\mathbf{M}$ made the major contribution to organize the patient data (symptoms and underlying diseases) for preliminary tables. Grönroos J performed the broth microdilution determinations and participated in writing the manuscript. Rantakokko-Jalava K took part in designing the study, helped to combine the susceptibility data with patient data, and reviewed the manuscript. All authors contributed substantially to the manuscript and have seen and approved the final version.

Funding Open Access funding provided by University of Helsinki including Helsinki University Central Hospital. Sihvonen received 1-month salary from HUS Diagnostic Center and 1-month grand form Finnish Society for Study of Infectious Diseases for this study. The funding organizations did not participate in any way in the study design, the data analysis, nor the writing of the manuscript.

Data Availability Not applicable.

Code availability Not applicable.

\section{Declarations}

Ethics approval The study was approved by Helsinki University Hospital (DOC130319-13032019094734) and the City of Helsinki (HEL 2019-004305).

Consent to participate Not applicable.

Consent for publication Not applicable.

Conflict of interest The authors declare no competing interests.

Open Access This article is licensed under a Creative Commons Attribution 4.0 International License, which permits use, sharing, adaptation, distribution and reproduction in any medium or format, as long as you give appropriate credit to the original author(s) and the source, provide a link to the Creative Commons licence, and indicate if changes were made. The images or other third party material in this article are included in the article's Creative Commons licence, unless indicated otherwise in a credit line to the material. If material is not included in the article's Creative Commons licence and your intended use is not permitted by statutory regulation or exceeds the permitted use, you will need to obtain permission directly from the copyright holder. To view a copy of this licence, visit http://creativecommons.org/licenses/by/4.0/.

\section{References}

1. S Narayanasamy K King A Dennison DW Spelman AK Aung 2017 Clinical characteristics and laboratory identification of Aerococcus infections: an Australian tertiary centre perspective Int J Microbiol 5684614. https://doi.org/10.1155/2017/5684614

2. Senneby E, Göransson L, Weiber S, Rasmussen MA (2016) A population-based study of aerococcal bacteraemia in the MALDI-TOF MS-era. Eur J Clin Microbiol Infect Dis 35:755762. https://doi.org/10.1007/s10096-016-2594-z

3. Sunnerhagen T, Nilson B, Olaison L, Rasmussen M (2016) Clinical and microbiological features of infective endocarditis caused by aerococci. Infection 44:167-173. https://doi.org/10. 1007/s15010-015-0812-8

4. Yasukawa K, Afzal Z, Mbang P, Stager CE, Musher DM (2014) Aerococcal infection at three US Tertiary Care Hospitals. South Med J 107:642-647. https://doi.org/10.14423/SMJ.0000000000 000158

5. Shelton-Dodge K, Vetter EA, Kohner PC, Nyre LM, Patel R (2011) Clinical significance and antimicrobial susceptibilities of Aerococcus sanguinicola and Aerococcus urinae. Diagn Microbiol Infect Dis 70:448-451. https://doi.org/10.1016/j. diagmicrobio.2010.09.001

6. de Jong MFC, Soetekouw R, ten Kate RW, Veenendaal D (2010) Aerococcus urinae: severe and fatal bloodstream infections and endocarditis. J Clin Microbiol 48:3445-3447. https://doi.org/10. 1128/JCM.00835-10

7. Senneby E, Petersson AC, Rasmussen M (2012) Clinical and microbiological features of bacteraemia with Aerococcus urinae. Clin Microbiol Infect 18:546-550. https://doi.org/10. 1111/j.1469-0691.2011.03609.x

8. Partridge L, Deelen J, Slagboom PE (2018) Facing up to the global challenges of ageing. Nature 561(7721):45-56. https:// doi.org/10.1038/s41586-018-0457-8

9. Rasmussen M (2016) Aerococcus: an increasingly acknowledged human pathogen. Clin Microbiol Infect 22:22-27. https:// doi.org/10.1016/j.cmi.2015.09.026

10. Opota O, Prod'hom G, Andreutti-Zaugg C, Dessauges M, Merz L, Greub G et al (2016) Diagnosis of Aerococcus urinae infections: importance of matrix-assisted laser desorption ionization time-of-flight mass spectrometry and broad-range 16S rDNA PCR. Clin Microbiol Infect 22(1):e1-e2. https://doi.org/10. 1016/j.cmi.2015.08.026

11. Lupo A, Guilarte YN, Droz S, Hirzel C, Furrer H, Endimiani A (2014) In vitro activity of clinically implemented $\beta$-lactams against Aerococcus urinae: presence of non-susceptible isolates in Switzerland. New Microbiol 37:563-566

12. Skov R, Cristensen JJ, Korner B, Frimodt-Moller N, Espersen F (2001) In vitro antimicrobial susceptibility of Aerococcus urinae to 14 antibiotics, and time-kill curves for penicillin, gentamicin and vancomycin. J Antimicrob Chemother 48:653-658. https://doi.org/10.1093/jac/48.5.653

13. EUCAST, The European Committee on Antimicrobial Susceptibility Testing. Breakpoint tables for interpretation of MICs and zone diameters. Version 10.0, 2020 (www.eucast.org).

14. Tai DBG, Go JR, Fida M, Saleh OA (2021) Management and treatment of Aerococcus bacteremia and endocarditis. Int $\mathbf{J}$ 
Infect Dis 102:584-589. https://doi.org/10.1016/j.ijid.2020.10. 096

15. Carkaci D, Nielsen XC, Fuursted K, Skov R, Skovgaard O, Trallero EP et al (2017) Aerococcus urinae and Aerococcus sanguinicola: susceptibility testing of 120 isolates to six antimicrobial agents using disk diffusion (EUCAST), Etest, and broth microdilution techniques. Open Microbiol J 11:160-166. https://doi.org/ $10.2174 / 1874285801711010160$

16. Humphries RM, Hindler JA (2014) In vitro antimicrobial susceptibility of Aerococcus urinae. J Clin Microbiol 52:2177-2180. https://doi.org/10.1128/JCM.00418-14

17. Sierra-Hoffman M, Watkins K, Jinadatha C, Fader R, Carpenter JL (2005) Clinical significance of Aerococcus urinae: a retrospective review. Diagn Microbiol Infect Dis 53(4):289-292. https://doi.org/ 10.1016/j.diagmicrobio.2005.06.021

18. Kays MB, Graff MA (2002) Broth microdilution and E-test for determining fluoroquinolone activity against Streptococcus pneumoniae. Ann Pharmacother 36:416-422. https://doi.org/10.1345/ aph.1A119

19. Azrad M, Tkhawkho L, Isakovich N, Nitzan O, Peretz A (2018) Antimicrobial susceptibility of Campylobacter jejuni and Campylobacter coli: comparison between Etest and a broth dilution method. Ann Clin Microbiol Antimicrob 17:23. https://doi.org/ 10.1186/s12941-018-0275-8
20. Hombach M, Böttger EC, Roos M (2013) The critical influence of the intermediate category on interpretation errors in revised EUCAST and CLSI antimicrobial susceptibility testing guidelines. Clin Microbiol Infect 19:169-171. https://doi.org/10.1111/ 1469-0691.12090

21. Cantón R, Livermore DM, Morosini MI, Díaz-Regañón J, Rossolini GM; PREMIUM Study Group (2017) Etest ${ }^{\circledR}$ versus broth microdilution for ceftaroline MIC determination with Staphylococcus aureus: results from PREMIUM, a European multicentre study. J Antimicrob Chemother 72:431-436. https://doi.org/10. 1093/jac/dkw442

22. Sauvage E, Kerff F, Terrak M, Ayala JA, Charlier P (2008) The penicillin-binding proteins: structure and role in peptidoglycan biosynthesis. FEMS Microbiol Rev 32:234-258. https://doi.org/ 10.1111/j.1574-6976.2008.00105.x

23. Oskooi M, Sunnerhagen T, Senneby E (2018) A prospective observational treatment study of aerococcal urinary tract infection. J Infect 76:354-360. https://doi.org/10.1016/j.jinf.2017.12. 009

Publisher's Note Springer Nature remains neutral with regard to jurisdictional claims in published maps and institutional affiliations. 